\title{
Endoscope-assisted fluorescence-guided resection allowing supratotal removal in glioblastoma surgery
}

\author{
Christoph Bettag, MD, ${ }^{1}$ Katharina Schregel, MD, ${ }^{2,3}$ Philip Langer, BSc, ${ }^{2}$ Carolina Thomas, MD, ${ }^{4}$ \\ Daniel Behme, MD, ${ }^{2}$ Christine Stadelmann, MD, ${ }^{4}$ Veit Rohde, MD, ${ }^{1}$ and Dorothee Mielke, MD ${ }^{1}$
}

Departments of ${ }^{1}$ Neurosurgery, ${ }^{2}$ Neuroradiology, and ${ }^{4}$ Neuropathology, Georg-August-University Göttingen; and ${ }^{3}$ Department of Neuroradiology, University Hospital Heidelberg, Germany

\begin{abstract}
OBJECTIVE Several studies have proven the benefits of a wide extent of resection (EOR) of contrast-enhancing tumor in terms of progression-free survival (PFS) and overall survival (OS) in patients with glioblastoma (GBM). Thus, grosstotal resection (GTR) is the main surgical goal in noneloquently located GBMs. Complete tumor removal can be almost doubled by microscopic fluorescence guidance. Recently, a study has shown that an endoscope with a light source capable of inducing fluorescence allows visualization of remnant fluorescent tumor tissue even after complete microscopic fluorescence-guided (FG) resection, thereby increasing the rate of GTR. Since tumor infiltration spreads beyond the borders of contrast enhancement on MRI, the aim of this study was to determine via volumetric analyses of the EOR whether endoscope-assisted FG resection enables supratotal resection beyond the borders of contrast enhancement.

METHODS The authors conducted a retrospective single-center analysis of a consecutive series of patients with primary GBM presumed to be noneloquently located and routinely operated on at their institution between January 2015 and February 2018 using a combined microscopic and endoscopic FG resection. A 20-mg/kg dose of 5-aminolevulinic acid (5-ALA) was administered 4 hours before surgery. After complete microscopic FG resection, the resection cavity was scanned using the endoscope. Detected residual fluorescent tissue was resected and embedded separately for histopathological examination. Nonenhanced and contrast-enhanced 3D T1-weighted MR images acquired before and within 48 hours after tumor resection were analyzed using 3D Slicer. Bias field-corrected data were used to segment brain parenchyma, contrast-enhancing tumor, and the resection cavity for volume definition. The difference between the pre- and postoperative brain parenchyma volume was considered to be equivalent to the resected nonenhancing but fluorescent tumor tissue. The volume of resected tumor tissue was calculated from the sum of resected contrast-enhancing tumor tissue and resected nonenhancing tumor tissue.
\end{abstract}

RESULTS Twelve patients with GBM were operated on using endoscopic after complete microscopic FG resection. In all cases, residual fluorescent tissue not visualized with the microscope was detected. Histopathological examination confirmed residual tumor tissue in all specimens. The mean preoperative volume of brain parenchyma without contrastenhancing tumor was $1213.2 \mathrm{~cm}^{3}$. The mean postoperative volume of brain parenchyma without the resection cavity was $1151.2 \mathrm{~cm}^{3}$, accounting for a mean volume of nonenhancing but fluorescent tumor tissue of $62.0 \mathrm{~cm}^{3}$. The mean relative rate of the overall resected volume compared to the contrast-enhancing tumor volume was $244.7 \%(p<0.001)$.

CONCLUSIONS Combined microscopic and endoscopic FG resection of GBM significantly increases the EOR and allows the surgeon to achieve a supratotal resection beyond the borders of contrast enhancement in noneloquently located GBM.

https://thejns.org/doi/abs/10.3171/2020.10.FOCUS20560

KEYWORDS 5-aminolevulinic acid; endoscope; fluorescence-guided surgery; glioblastoma

$\mathrm{T}$ HE survival of patients with glioblastoma (GBM) remains poor despite recent advances. A greater extent of resection (EOR) has been associated with better progression-free survival (PFS) and overall survival (OS) in several studies. ${ }^{1-3}$ If feasible, gross-total resection (GTR) of contrast-enhancing tumor tissue, as seen on preoperative MRI, is one of the main surgical goals. The use of 5-aminolevulinic acid (5-ALA) and microscopic fluorescence-guided (FG) resection increases the rate of GTR from $36 \%$ to $65 \%{ }^{4}$ However, microscopic FG resection is still limited, as fluorescence depends on various factors such as cell density and cellular metabolism. ${ }^{5}$ One

ABBREVIATIONS 5-ALA = 5-aminolevulinic acid; EOR = extent of resection; FG = fluorescence-guided; GBM = glioblastoma; GTR = gross-total resection; OS = overall survival; PFS = progression-free survival. 
of the major factors influencing the detectability of tumor by fluorescence is adequate exposure to blue light. A recently published study has shown that the assistance of an endoscope with a white and a blue light source, which is capable of inducing fluorescence, allows one to further increase the percentage of GTR by overcoming the limitations of the microscope. ${ }^{6}$ However, the impact of combined microscopic and endoscopic FG resection on EOR has not been quantified. As previous studies have shown that malignant tumor cells spread beyond the areas of contrast enhancement on MRI, ${ }^{7-9}$ the aim of our study was to determine if endoscope-assisted FG resection enables supratotal resection beyond the borders of contrast-enhancing GBM by comparing the volume of contrast-enhancing tumor on preoperative MRI with the volume of resected GBM tissue on postoperative MRI after such a resection.

\section{Methods}

We performed a retrospective single-center analysis of a consecutive series of patients with primary GBM presumed to be noneloquently located and routinely operated on in our department between January 2015 and February 2018 using combined microscopic and endoscopic FG resection. Volume measurements were performed by experienced neuroradiologists. The study was reviewed by the local ethics committee. All patients were informed about the intraoperative use of the endoscope. Written consent was obtained from all patients within the standard informed consent process for surgery.

\section{Inclusion and Exclusion Criteria}

Only those patients with data sets containing 3D T1weighted MR images before and after administration of a contrast agent $(0.1 \mathrm{mmol} / \mathrm{ml}$ gadobutrol per $\mathrm{kg}$ body weight, Gadovist, Bayer Vital GmbH) pre- and post-tumor resection were included. Patients were excluded if the tumor did not reach the brain surface in order to avoid a bias of the volume measurements in case of brain tissue removal within the approach corridor. Recurrent GBM was also an exclusion criterion because it was not possible to distinguish without question contrast-enhancing tumor from postoperative and postradiation alterations, which would have been a potential source of error in the measurement of the preoperative tumor volume.

\section{Surgical Protocol}

A standard $20-\mathrm{mg} / \mathrm{kg}$ dose of 5-ALA was given 4 hours prior to surgery. A standard microscopic FG resection (OPMI Pentero 800, Carl Zeiss) was performed. Neuronavigation based on the preoperatively acquired, contrast-enhanced, 3D, T1-weighted MRI data set was used in all patients (VectorVision Sky navigation system and Brainlab software, Brainlab). Since all lesions were presumed to be in a noneloquent location, cortical mapping or intraoperative monitoring was considered unnecessary. After complete microscopic removal of all fluorescent tissue (solid and vague), the resection cavity was scanned using an endoscope (Hopkins II, $4 \mathrm{~mm}$, viewing angle $0^{\circ}$, Karl Storz) with a special light source (D-Light C, Karl Storz) and camera system (Tricam SL II, Karl Storz). The
D-Light allows switching between the white light and the blue light source mode by means of an appropriate band-pass filter in the light transmission path. A long-pass filter at the eyepiece of the endoscope blocks the excitation light, which enables detection of fluorescence signals from the tumor cells. The excitation and detection filter system allows enough blue light to be transmitted so that the red fluorescence from the endogenous fluorochromes and nonspecific protoporphyrin IX (PpIX) fluorescence is suppressed, causing the normal tissue to be visualized as blue..$^{10}$ Microscopic fluorescent tissue and endoscopic fluorescent tissue that had not been visualized by the microscope were completely removed and embedded separately for histopathological examination. All patients underwent MRI within 48 hours after surgery.

\section{Image Analysis Protocol}

MRI data acquired in a routine clinical setting were used and retrieved from the hospital's PACS. Data were analyzed using 3D Slicer (version 4.10.1 for Windows, www.slicer.org $\left.{ }^{11}\right)$. For whole-brain volumetry, nonenhanced 3D T1-weighted data sets acquired before and after surgery were used. After bias field correction, brain parenchyma was segmented using the Swiss Skull Stripper extension available via Slicer's extension manager, and label maps were manually corrected as appropriate. Additionally, CSF-containing spaces were removed from the masks using dedicated in-house software. Bias fieldcorrected, contrast-enhanced, 3D, T1-weighted data sets pre- and postsurgery were used for volumetric analyses of the tumor and the resection cavity, respectively. Tumor and cavity were segmented semiautomatically by applying Slicer's Grow Cut Effect method, and masks were manually corrected if needed. Residual tumor was defined as any visible contrast enhancement $>0.175 \mathrm{~cm}^{3}$ on postoperative MRI. The following parameters were derived using dedicated in-house software: volume of contrast-enhancing tumor on preoperative MRI, volume of resection cavity on postoperative MRI, preoperative volume of brain parenchyma without tumor, and postoperative volume of brain parenchyma without the resection cavity. The difference in the volume of brain parenchyma before and after tumor resection was calculated to yield the volume of resected nonenhancing tissue. The volume of the overall resected tissue was then calculated as the sum of the contrast-enhancing tumor volume plus the volume of the nonenhancing tissue. The EOR, expressed as a percentage, was calculated as follows: [(contrast-enhancing tumor volume + nonenhancing tissue)/preoperative tumor volume] $\times 100$.

\section{Statistical Analysis}

Statistical analysis was performed using SPSS (version 23, IBM Corp.). Descriptive statistics were used for the assessment of patient characteristics. Mean values and standard deviations were calculated for the volume measurements. The volume of the overall resected tissue was also related to the preoperatively assessed tumor volume in terms of percentage. The tumor volume was compared to the overall resected tissue and the resection cavity using 
an unpaired t-test. A Pearson correlation was performed for tumor volume and the volume of overall resected tumor tissue. A p value $<0.05$ was considered statistically significant.

\section{Results}

Twenty-three patients with primary GBM had been operated on using microscopic as well as endoscopic FG resection. Twelve patients ( 8 male, 4 female) with primary GBM were included in our analysis; 4 patients with recurrent GBM and 7 patients with deep-seated GBM were excluded. The mean age was 65 (range 47-77) years. Tumor location was frontal in 6 patients, temporal in 5 patients, and parietal in 1 patient (Table 1). In 7 patients, O6-methylguanine-DNA methyltransferase (MGMT) promoter was methylated. In all patients, residual fluorescent tissue at the margin of the resection cavity was detected by the endoscope after completed microscopic FG resection (Fig. 1). That fluorescent tissue was completely resected. All patients had complete tumor resection without residual contrast-enhancing tumor on early postoperative MRI.

\section{Volume Measurements}

The mean $( \pm \mathrm{SD})$ preoperative volume of brain parenchyma without contrast-enhancing tumor tissue and CSF was $1213.2 \mathrm{~cm}^{3}\left( \pm 98.5 \mathrm{~cm}^{3}\right)$. The mean postoperative volume of the brain parenchyma without the resection cavity and CSF was $1151.2 \mathrm{~cm}^{3}\left( \pm 106.3 \mathrm{~cm}^{3}\right)$. The mean contrast-enhancing tumor volume was $52.2 \mathrm{~cm}^{3}\left( \pm 31.8 \mathrm{~cm}^{3}\right)$. The mean volume of the resection cavity was $32.9 \mathrm{~cm}^{3}( \pm$ $\left.21.1 \mathrm{~cm}^{3}\right)$. The mean volume of the nonenhancing tumor tissue was $62.0 \mathrm{~cm}^{3}\left( \pm 46.1 \mathrm{~cm}^{3}\right)$, and the volume of the overall resected fluorescent tissue was $114.2 \mathrm{~cm}^{3}( \pm 70.0$ $\mathrm{cm}^{3}$; Table 1).

\section{Extent of Resection}

In 9 of 12 patients (75\%), the resection cavity was smaller than the contrast-enhancing tumor (Table 1). The mean volume of the resection cavity was significantly smaller than the contrast-enhancing tumor volume $(95 \%$ CI $4.89-35.99, \mathrm{p}=0.015)$. There was no correlation between the tumor volume and the volume of the resection cavity (Pearson correlation coefficient $\mathrm{r}=0.21$, 95\% CI -0.527 to $0.800, p=0.513$; Fig. 2). The volume measurements showed a significantly larger EOR on postoperative MRI compared to the mean preoperative contrast-enhancing tumor volume (95\% CI 32.49-91.24, $\mathrm{p}<0.001)$. In all patients $(100 \%)$, the EOR was greater than the contrastenhancing tumor volume (Fig. 3). The correlation between the contrast-enhancing tumor volume and the overall resected volume was excellent (Pearson correlation coefficient $\mathrm{r}=0.851,95 \%$ CI $0.332-0.962, \mathrm{p}<0.001)$. The mean relative size of the overall resected volume compared to the tumor volume was $244.7 \%$ ( $\pm 102.2 \%)$.

\section{Histopathological Findings}

Twenty-four microscopic fluorescent specimens and 32 endoscopic fluorescent specimens were collected. Twelve of the microscopic fluorescent specimens from the central

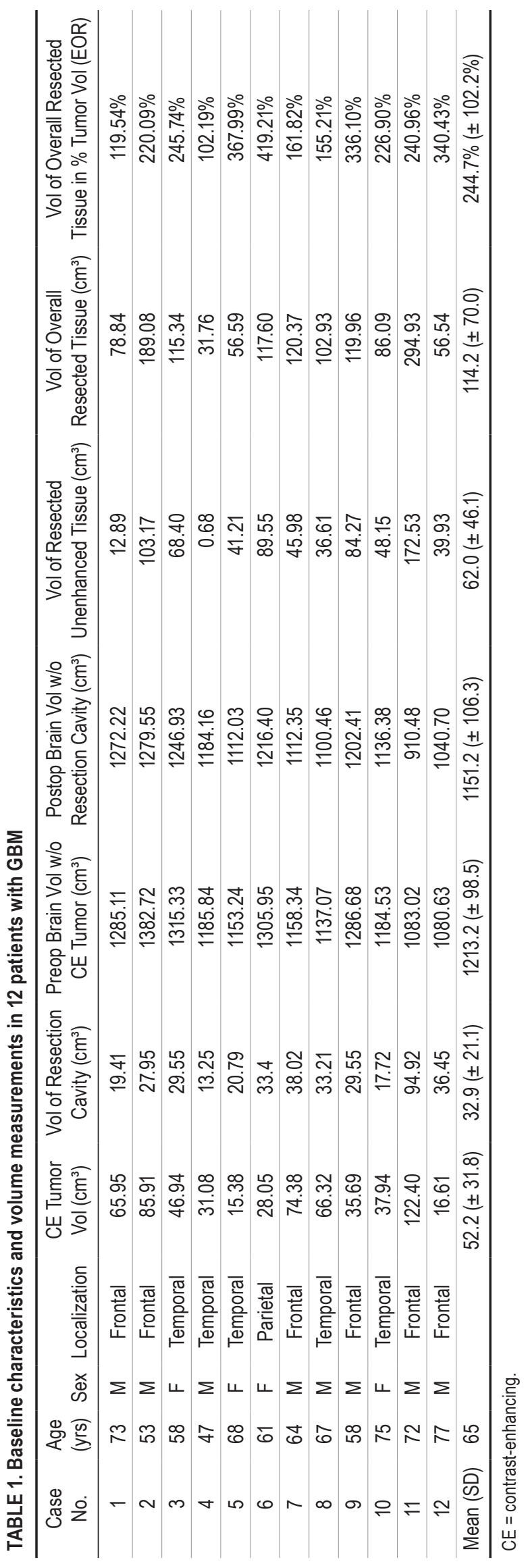

Neurosurg Focus Volume 50 • January 2021 

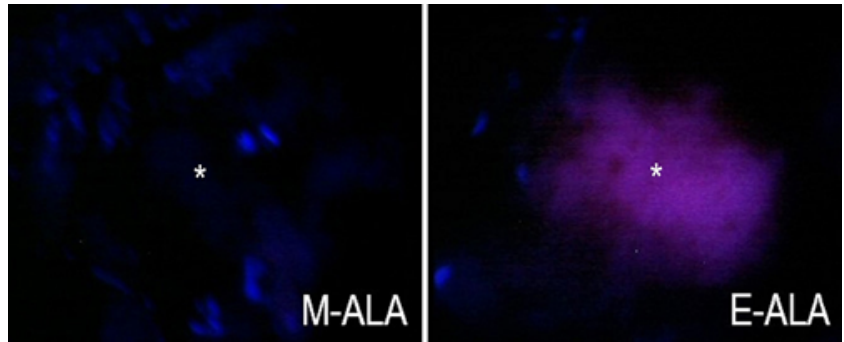

FIG. 1. Views of the resection site through the microscope (M-ALA, left) and the endoscope (E-ALA, right). The same tissue visualized by the microscope or the endoscope is marked by asterisks. No residual fluorescent tissue is visible after completed microscopic FG resection (M-ALA). After using the endoscope with a blue light source, the tissue at the margin of the resection cavity becomes fluorescent (E-ALA). Histopathological examination of the biopsies taken from the residual fluorescent area confirmed tumor tissue.

tumor area showed solid tumor tissue, whereas 12 of the microscopic specimens from the tumor margin and all of the endoscopic specimens showed brain tissue diffusely infiltrated by tumor (Fig. 4).

\section{Postoperative Course}

Preoperatively, 3 patients had presented with slight arm paresis caused by edema. Four patients had experienced preoperative seizures. No new or worsened neurological deficits were recorded after surgery. All preexisting neurological deficits improved under oral steroids during hospitalization. No postoperative seizures occurred. The mean Karnofsky Performance Status (KPS) at discharge was $90.8 \%( \pm 12.4 \%)$. The mean length of stay was $9.7( \pm$ 1.1) days.

\section{Discussion}

Combined microscopic and endoscopic FG resection enables the surgeon to achieve supratotal resection beyond the borders of contrast enhancement in GBM surgery. The overall resected tumor volume was significantly larger than the contrast-enhancing tumor volume on MRI. In all cases, residual fluorescent tissue was detected by the endoscope that had not been visualized by the microscope. Histopathological examination confirmed variable infiltration of tumor cells in the separately preserved endoscopic biopsies.

\section{Impact on EOR}

After publication of a technical note in 2014, a first clinical series showed that residual fluorescent tumor tissue not visualized with the microscope could be detected with endoscopic assistance. ${ }^{6,10}$ This finding is not surprising because inducing fluorescence is mainly related to adequate exposure to blue light. According to the inversesquare law ("illumination of a surface receiving its flux from a point source is inversely proportional to the square of the distance between the surface and the source"), the endoscope, as compared to the microscope, is capable of significantly reducing the distance between the light source and the tissue. ${ }^{6}$ Besides the barriers of the depth of the surgical field, the presence of overhanging tissue or collapse of the resection cavity can lead to insufficient visualization of the tumor margins. ${ }^{12}$ Furthermore, blind spots for microscopic FG resection can exist in the area of the craniotomy ${ }^{10}$ as well as at the margin of the tumor due to collapse of the resection cavity. ${ }^{6}$ These blind spots can be visualized with the endoscope. Thus, endoscopic assistance appears to be a useful adjunct to increase the radicality of resection in GBM surgery. ${ }^{6}$

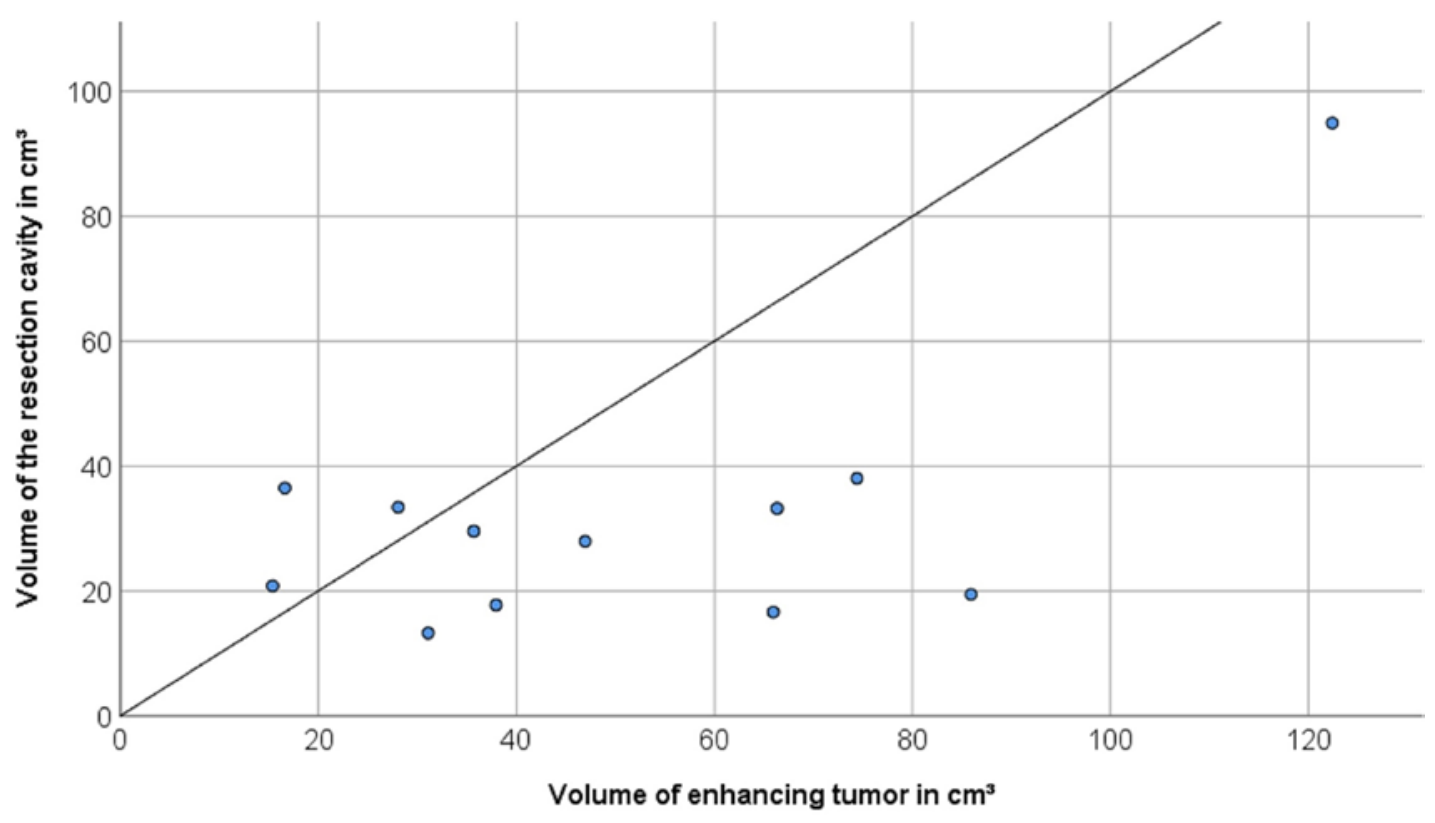

FIG. 2. The resection cavity was smaller than the contrast-enhancing tumor volume in 9 of 12 cases. There was no correlation between the volume of contrast-enhancing tumor and the resection cavity (Pearson correlation coefficient $r=0.21,95 \% \mathrm{Cl}-0.527$ to $0.800, p=0.513)$. 


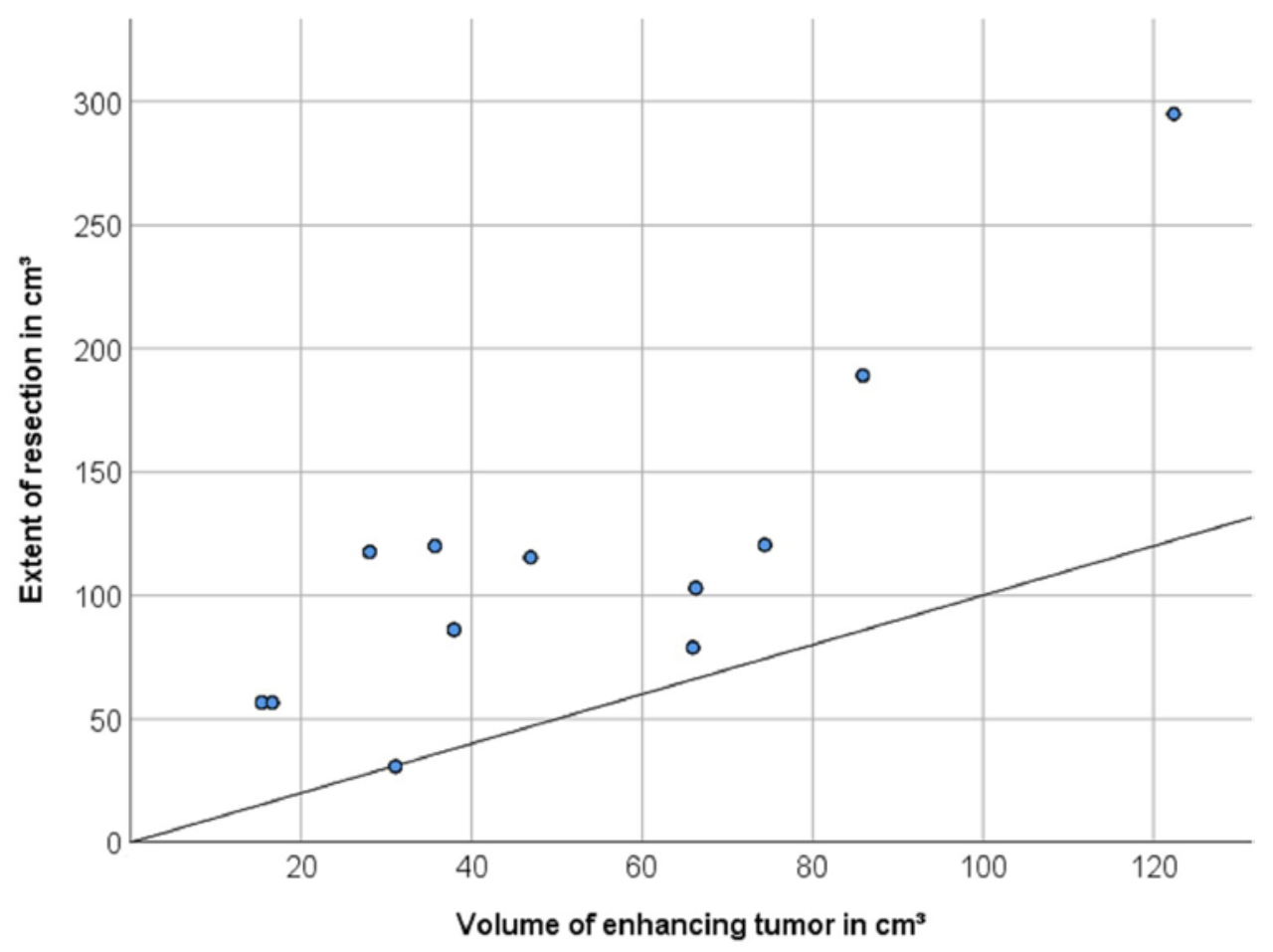

FIG. 3. The EOR was at least $101 \%$ compared to the contrast-enhancing tumor volume, and in 8 of 12 cases it was greater than $200 \%$. The correlation between the contrast-enhancing tumor volume on preoperative MRI and the overall resected volume on postoperative MRI was excellent (Pearson's correlation coefficient $r=0.851, p=0.00045$ ).

Whether combined microscopic and endoscopic FG resection is truly superior to microscopic FG resection must be defined in a prospective comparative study. Schucht et al. showed similar results for the mean EOR (242\%) using microscopic FG resection in a small retrospective cohort. ${ }^{13}$ However, segmentations were not performed by neuroradiologists in that study. Furthermore, the SD of the mean EOR was 4 times higher than that in our cohort, suggesting very high heterogeneity of the EOR in their cohort. In fact, the EOR remained lower than $200 \%$ in half of the patients in that cohort, whereas only 33\% of the patients in the present study had such an EOR. Taking this into account, it can be suggested that the combined use of a microscope and an endoscope may be superior to the use of the microscope alone in terms of visualizing residual fluorescent tissue, thereby increasing the EOR substantially.

\section{Volumetric Measurements}

As our volumetric analysis showed, the EOR was significantly larger $(245 \%)$ than the tumor volume by using endoscope-assisted FG resection. As previous studies have confirmed, EOR can be increased by FG resection ${ }^{4}$ or intraoperative MRI (iMRI) ${ }^{14,15}$ compared to that obtained with standard neuronavigated microscopic resection. However, whether supratotal resection was achieved in these studies remains unclear, as only residual tumor volume, not the volume of the EOR, was quantified. Despite the unquestionable advantages of iMRI regarding an increased rate of GTR, ${ }^{14-17}$ its high costs in terms of money and time are reasons for its reduced availability in neurosurgical centers..$^{18-20}$ Therefore, FG resection is mostly performed in Europe. As mentioned before, fluorescent tissue is mainly dependent on adequate exposure to blue light, which can be limited using microscopic fluorescence guidance. The endoscopic assistance appears very useful to increase the EOR in GBM surgery. ${ }^{6}$

Recently published studies have suggested that the enhancing tumor volume underestimates the full extent of the tumor burden since tumor infiltration spreads beyond the borders of contrast enhancement, which are characteristically hyperintense on FLAIR sequences. ${ }^{21-23} \mathrm{We}$ did not perform volumetric analyses of the resected hyperintensity parts on the FLAIR sequences because of missing 3D data sets. Future studies should investigate whether the EOR of FLAIR hyperintense lesions can be increased by endoscopic assistance.

\section{Impact on OS}

Several studies have shown a benefit of GTR compared with subtotal resection in GBM surgery. ${ }^{1,2,14,21}$ A study by Esquenazi et al. showed a significant benefit from supratotal resection with a mean OS of 34.2 months. ${ }^{8} \mathrm{~A}$ benefit in OS has also been demonstrated when fluorescent tissue is completely removed, even if residual tumor is not displayed as contrast enhancement on MRI. ${ }^{1}$ As these data suggest and as most of the recurrences appear at or near the resection cavity, ${ }^{24}$ supratotal resection beyond the borders of contrast enhancement may be beneficial in GBM surgery. Since our cohort is small and selective, the impact 


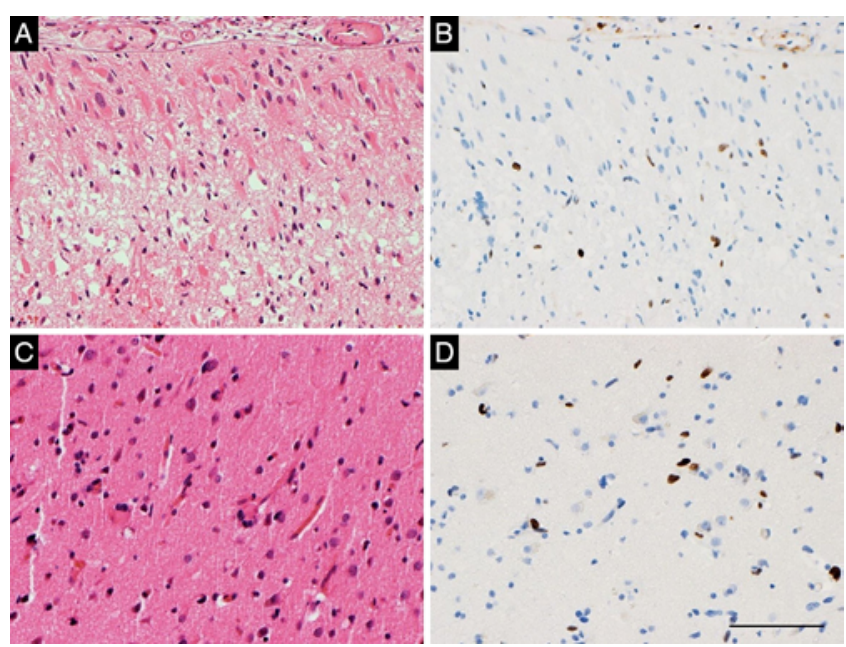

FIG. 4. Histopathological examination of E-ALA-positive tumor samples (undetected by M-ALA) showed infiltration of tumor-adjacent gray matter by neoplastic GBM cells. H \& E staining of an M-ALA-positive tumor sample (A) shows complete replacement of the cortical neuronal layers by GBM cells, best detected by immunostaining with the proliferation marker Ki-67 (B). Gray matter with a discrete increase in cell density in an E-ALA-positive sample (C), undetected by M-ALA, with moderate infiltration by GBM cells (D) immunolabeled with Ki-67. Bar $=100 \mu \mathrm{m}$.

on OS was not quantified because it would be inconclusive. A benefit of endoscope-assisted FG resection in OS has to be confirmed in a prospective study with a larger cohort.

\section{Study Strengths and Limitations}

Our study has several limitations. Our cohort is small and selective. All tumors were easy to access since they reached the brain surface and were located in noneloquent areas. Although the advantage of endoscopic assistance may especially apply for deeper-seated lesions, given increasingly insufficient illumination of the resection cavity by the microscope, these lesions were excluded since the eventually necessary removal of healthy brain tissue within the approach corridor would have biased the volume measurements. Furthermore, the value of endoscopic assistance is limited in tumors that are located in eloquent areas because supramaximal resection will result in neurological deficits and is therefore avoided. Still, the feasibility, safety, and possible benefit of a combined endoscopeguided tumor resection and intraoperative monitoring in an eloquently located lesion could be the subject of further studies.

Our volumetric measurements were indirect, and the volume of the resected tissue, which was additionally resected with the assistance of the endoscope, could not be quantified. Furthermore, changes in cerebral blood volume as well as edema volume after tumor resection could not be quantified. Hence, the results of this study must be interpreted with care. Since all patients received perioperative steroids, a reduction in peritumoral edema might lead to incorrect volumetric measurements of the EOR. However, we believe that the impact of this possible bias is low, as all preoperative MRI studies were performed within 3 days prior to surgery, and all postoperative MRI studies within
48 hours after surgery. As residual fluorescent tumor tissue was detected in all cases after completed microscopic FG resection, we believe that endoscopic assistance is a useful adjunct in GBM surgery.

\section{Conclusions}

Endoscope-assisted FG resection of GBM significantly increases the EOR and allows the surgeon to achieve a supratotal resection beyond the borders of contrast enhancement in noneloquently located GBM. Residual fluorescent tumor tissue at the margins of the resection cavity, not previously visualized by a microscope, can be detected by an endoscope, indicating that the assistance of the endoscope allows supramaximal resection by visualizing the zone of tumor infiltration. A possible benefit in PFS and OS still has to be determined in a larger cohort.

\section{References}

1. Aldave G, Tejada S, Pay E, et al. Prognostic value of residual fluorescent tissue in glioblastoma patients after gross total resection in 5-aminolevulinic acid-guided surgery. Neurosurgery. 2013;72(6):915-921.

2. Brown TJ, Brennan MC, Li M, et al. Association of the extent of resection with survival in glioblastoma: a systematic review and meta-analysis. JAMA Oncol. 2016;2(11):1460-1469.

3. Sanai N, Polley MY, McDermott MW, et al. An extent of resection threshold for newly diagnosed glioblastomas. $J$ Neurosurg. 2011;115(1):3-8.

4. Stummer W, Pichlmeier U, Meinel T, et al. Fluorescenceguided surgery with 5-aminolevulinic acid for resection of malignant glioma: a randomised controlled multicentre phase III trial. Lancet Oncol. 2006;7(5):392-401.

5. Collaud S, Juzeniene A, Moan J, Lange N. On the selectivity of 5-aminolevulinic acid-induced protoporphyrin IX formation. Curr Med Chem Anticancer Agents. 2004;4(3):301-316.

6. Bettag C, Hussein A, Behme D, Maragkou T, Rohde V, Mielke D. Endoscopic fluorescence-guided resection increases radicality in glioblastoma surgery. Oper Neurosurg (Hagerstown). 2020;18(1):41-46.

7. Eidel O, Burth S, Neumann JO, et al. Tumor infiltration in enhancing and non-enhancing parts of glioblastoma: a correlation with histopathology. PLoS One. 2017;12(1):e0169292.

8. Esquenazi Y, Friedman E, Liu Z, et al. The survival advantage of "supratotal" resection of glioblastoma using selective cortical mapping and the subpial technique. Neurosurgery. 2017;81(2):275-288.

9. Kelly PJ, Daumas-Duport C, Kispert DB, et al. Imagingbased stereotaxic serial biopsies in untreated intracranial glial neoplasms. J Neurosurg. 1987;66(6):865-874.

10. Rapp M, Kamp M, Steiger HJ, Sabel M. Endoscopic-assisted visualization of 5-aminolevulinic acid-induced fluorescence in malignant glioma surgery: a technical note. World Neurosurg. 2014;82(1-2):e277-e279.

11. Fedorov A, Beichel R, Kalpathy-Cramer J, et al. 3D Slicer as an image computing platform for the Quantitative Imaging Network. Magn Reson Imaging. 2012;30(9):1323-1341.

12. Morshed RA, Han SJ, Lau D, Berger MS. Wavelengthspecific lighted suction instrument for 5-aminolevulinic acid fluorescence-guided resection of deep-seated malignant glioma: technical note. J Neurosurg. 2018;128(5):1448-1453.

13. Schucht P, Knittel S, Slotboom J, et al. 5-ALA complete resections go beyond MR contrast enhancement: shift corrected volumetric analysis of the extent of resection in surgery for glioblastoma. Acta Neurochir (Wien). 2014;156(2): 305-312. 
14. Kuhnt D, Becker A, Ganslandt O, et al. Correlation of the extent of tumor volume resection and patient survival in surgery of glioblastoma multiforme with high-field intraoperative MRI guidance. Neuro Oncol. 2011;13(12):1339-1348.

15. Senft C, Bink A, Franz K, et al. Intraoperative MRI guidance and extent of resection in glioma surgery: a randomised, controlled trial. Lancet Oncol. 2011;12(11):997-1003.

16. Hatiboglu MA, Weinberg JS, Suki D, et al. Impact of intraoperative high-field magnetic resonance imaging guidance on glioma surgery: a prospective volumetric analysis. Neurosurgery. 2009;64(6):1073-1081.

17. Knauth M, Wirtz CR, Tronnier VM, et al. Intraoperative MR imaging increases the extent of tumor resection in patients with high-grade gliomas. AJNR Am J Neuroradiol. 1999; 20(9):1642-1646.

18. Jankovski A, Francotte F, Vaz G, et al. Intraoperative magnetic resonance imaging at 3-T using a dual independent operating room-magnetic resonance imaging suite: development, feasibility, safety, and preliminary experience. Neurosurgery. 2008;63(3):412-426.

19. Martin XP, Vaz G, Fomekong E, et al. Intra-operative 3.0 T magnetic resonance imaging using a dual-independent room: long-term evaluation of time-cost, problems, and learningcurve effect. Acta Neurochir Suppl. 2011;109:139-144.

20. Schneider JP, Trantakis C, Rubach M, et al. Intraoperative MRI to guide the resection of primary supratentorial glioblastoma multiforme-a quantitative radiological analysis. Neuroradiology. 2005;47(7):489-500.

21. Grabowski MM, Recinos PF, Nowacki AS, et al. Residual tumor volume versus extent of resection: predictors of survival after surgery for glioblastoma. J Neurosurg. 2014;121(5): $1115-1123$.

22. Grossman R, Shimony N, Shir D, et al. Dynamics of FLAIR volume changes in glioblastoma and prediction of survival. Ann Surg Oncol. 2017;24(3):794-800.

23. Marko NF, Weil RJ, Schroeder JL, et al. Extent of resection of glioblastoma revisited: personalized survival modeling facilitates more accurate survival prediction and supports a maximum-safe-resection approach to surgery. J Clin Oncol. 2014;32(8):774-782
24. Lemée JM, Clavreul A, Menei P. Intratumoral heterogeneity in glioblastoma: don't forget the peritumoral brain zone. Neuro Oncol. 2015;17(10):1322-1332.

\section{Disclosures}

The authors report no conflict of interest concerning the materials or methods used in this study or the findings specified in this paper.

\section{Author Contributions}

Conception and design: Bettag, Rohde, Mielke. Acquisition of data: Bettag, Schregel, Langer, Thomas, Behme, Stadelmann, Mielke. Analysis and interpretation of data: Bettag. Drafting the article: Bettag. Critically revising the article: all authors. Reviewed submitted version of manuscript: all authors. Approved the final version of the manuscript on behalf of all authors: Bettag. Statistical analysis: Bettag. Administrative/technical/ material support: Schregel, Langer, Thomas, Behme, Stadelmann, Mielke. Study supervision: Rohde, Mielke.

\section{Supplemental Information}

\section{Previous Presentations}

Portions of this work were presented at the annual meeting of the German Society of Neurosurgery held in Lübeck, Germany, on June 21-24, 2020.

\section{Correspondence}

Christoph Bettag: Georg-August-University Göttingen, Germany. christoph.bettag@med.uni-goettingen.de. 